\title{
STEADY TIME-HARMONIC OSCILLATIONS IN A LINEAR THERMOELASTIC PLATE MODEL
}

BY

PETER SCHIAVONE (Dept. of Mechanical Engineering, Univ. of Alberta, Edmonton, Alberta, Canada)

AND

R. J. TAIT (Department of Mathematics, University of Alberta, Edmonton, Alberta, Canada)

\begin{abstract}
We examine the bending of a Mindlin-type thermoelastic plate when the source terms are time-harmonic with angular frequency $\omega$, and sufficient time has elapsed for the system to have reached a steady-state. We show that in an infinite plate the solution can be represented as the sum of five waves all but one of which exhibit damping. By formulating appropriate radiation conditions we prove uniqueness results for exterior boundary value problems subject to certain regularity assumptions and a condition on the angular frequency of oscillation.
\end{abstract}

1. Introduction. In time-harmonic problems for the wave equation [1]:

$$
c^{2} \Delta u+f(x, t)=\frac{\partial^{2} u}{\partial t^{2}} \quad\left(t>0, x \in \mathbf{R}^{2}\right)
$$

(here $f$ is a vector of sources and $u$ is the displacement vector), the time dependence of all sources of disturbance is assumed to be harmonic. That is, they may be represented in the form

$$
f(x, t)=\operatorname{Re}\left[F(x) e^{-i w t}\right]
$$

where $F$ may be a complex-valued function of position. It is now reasonable to assume that the solution takes the form

$$
u(x, t)=\bar{u}(x, t)+\operatorname{Re}\left[U(x) e^{-i w t}\right]
$$

where again, $U$ may be complex-valued. The first term on the right-hand side of (1.3) is called the transient solution and the second term the steady-state solution. The initial conditions influence the system only through the transient part while the boundary conditions are assigned to $U(x)$. We now assume that a sufficiently long period of time has elapsed so that the transient part $\bar{u}(x, t)$ has vanished [2]. Hence, if we are interested in the response of the system long after the monochromatic excitation began to act, the steady-state part constitutes the solution no matter what 
the initial conditions. The initial-boundary value problem is then replaced by a boundary value problem that simplifies the analysis.

The study of such "steady-state" solutions for (1.1) leads to the well-known problems for the reduced wave equation (see, for example, [3]).

In this paper we study steady-state solutions of thermoelastic plate bending by applying the above reasoning to the linear thermoelastic thin plate model introduced in [4]. As in the case of (1.1), the simplifications leading to the reduced wave equations of thermoelastic bending lead, in certain cases, to a loss of uniqueness because of the appearance of proper oscillation frequencies. In the case of exterior boundary value problems for the reduced wave equation from (1.1), uniqueness is usually guaranteed by imposing the Sommerfeld radiation condition [1]. A similar approach is taken by Kupradze in [5] for the general equations of elasticity and thermoelasticity.

The equations considered here, however, display characteristics not previously encountered in the literature. The main difficulties arise when we try to apply Helmholtz's theorem to a solution of the bending equations in the exterior domain. The decomposition is not as straightforward as in classical thermoelasticity [5]. Only one part satisfies a Helmholtz equation so that we can impose only one Sommerfeld-type radiation condition. Nevertheless, with repeated applications of Rellich's Lemma and standard results from potential theory [3], we prove uniqueness results for corresponding boundary value problems of the Dirichlet, Neumann, and mixed-type for the bending of an infinite thermoelastic thin plate with a hole.

Steady time-harmonic oscillations are an important state in their own right, frequently occurring in practical applications, but also very significant in the study of full dynamic problems [5].

The thin plate theory on which our model is based is presented in [6] and compared to classical plate theory, in the case of flexural waves, in [7].

Throughout the paper Greek and Latin subscripts take the values 1, 2 and 1, 2, 3 , respectively, summation is carried out over repeated indices, $x=\left(x_{1}, x_{2}\right)$ and $x=\left(x_{1}, x_{2}, x_{3}\right)$ are generic points referred to orthogonal Cartesian coordinates in $\mathbf{R}^{2}$ and $\mathbf{R}^{3}$, respectively, a superscript $\mathrm{T}$ indicates matrix transposition, $(\cdots)_{\alpha}=$ $\partial(\cdots) / \partial x_{\alpha}, \Delta$ is the Laplacian, and $\delta_{i j}$ is the Kronecker delta. For simplicity we use the same symbol to indicate both a point and a position vector in $\mathbf{R}^{2}$. Also, vector functions are not distinguished from scalar ones, their nature being obvious from the context.

2. Basic formulation. Let $\bar{S} \times\left[-\frac{h_{0}}{2}, \frac{h_{0}}{2}\right]$ be the region occupied by a homogeneous thin elastic plate, where $S$ is a domain in $\mathbf{R}^{2}$ bounded by a simple closed $C^{2}$-curve $\partial S$ and $0<h_{0}=$ const. $\ll \operatorname{diam} S$ is the thickness. We assume that, in addition to mechanical loads, the plate is subject to an unknown temperature distribution $\tau\left(x_{1}, x_{2}, x_{3}, t\right)$ ( $t$ is time) measured from a reference state of uniform temperature distribution $\tau_{0}$, at which temperature the plate is free from thermal stresses and strains. It is further assumed that the plate is elastically and thermally isotropic. 

[4]:

The equations of motion for the bending of a Mindlin-type thermoelastic plate are

$$
\begin{gathered}
h^{2}(\lambda+\mu) \operatorname{grad} \operatorname{div} u+\mu\left(h^{2} \Delta u-u-\operatorname{grad} u_{3}\right)-\operatorname{grad} u_{4}=\rho h^{2} \frac{\partial^{2} u}{\partial t^{2}}-H, \\
\mu\left(\Delta u_{3}+\operatorname{div} u\right)=\rho \frac{\partial^{2} u_{3}}{\partial t^{2}}-F_{3}, \\
\Delta u_{4}-\frac{1}{K} \frac{\partial u_{4}}{\partial t}-\eta \alpha h^{2}(3 \lambda+2 \mu) \frac{\partial}{\partial t} \operatorname{div} u=N .
\end{gathered}
$$

Here we have $u=u\left(x_{\alpha}, t\right)=\left(u_{1}, u_{2}, 0\right)^{\mathrm{T}} ; u_{3}=u_{3}\left(x_{\alpha}, t\right) ; u_{4}=u_{4}\left(x_{\alpha}, t\right)=$ $\frac{(3 \lambda+2 \mu)}{h_{0}} \int_{-h_{0} / 2}^{h_{0} / 2} x_{3} \varepsilon_{\tau} d x_{3} ; H=H\left(x_{\alpha}, t\right)=\left(H_{1}, H_{2}, 0\right)^{\mathrm{T}} ; F_{3}=F_{3}\left(x_{\alpha}, t\right) ; N=N\left(x_{\alpha}, t\right)$; $h^{2}=h_{0}^{2} / 12 ; \lambda$ and $\mu$ are the Lamé constants; $\varepsilon_{\tau}$ denotes the thermal strain; $\alpha$ is the coefficient of thermal expansion; $\eta=(2 \mu+3 \lambda) \alpha \tau_{0} / \lambda_{0} ; K=\lambda_{0} /(\rho c) ; \rho$ and $c$ are, respectively, the mass density and specific heat of the plate, and $\lambda_{0}>0$ is the (constant) coefficient of thermal conductivity. It should be noted that $H$ and $F_{3}$ characterize resultant body forces and couples, and forces and couples on the plate's faces; $N$ is a known quantity that represents heat generation within the plate and measurements of temperatures of the faces and in the surrounding medium. In accordance with the plate assumptions [4] $u_{i}$ characterize displacement and $u_{4}$ the resultant "thermal moment" on the plate's middle surface.

In what follows we assume that

$$
\rho>0, \quad c>0, \quad \alpha>0, \quad \mu>0, \quad 3 \lambda+2 \mu>0, \quad \tau_{0}>0 .
$$

Suppose now that all source terms are separable with respect to space and time and that the time dependency is periodic, e.g.,

$$
H\left(x_{\alpha}, t\right)=\operatorname{Re}\left[m\left(x_{\alpha}\right) e^{-i \omega t}\right],
$$

where $\omega \in \mathbf{R}^{+}$is the frequency of oscillation and $m=m^{(1)}+i m^{(2)}$ is some complexvalued vector function. If sufficient time has elapsed for the transient part of any solution to $(2.1)-(2.3)$ to have vanished, we can also write

$$
\begin{aligned}
& u_{i}\left(x_{\alpha}, t\right)=\operatorname{Re}\left[v_{i}\left(x_{\alpha}\right) e^{-i \omega t}\right], \\
& u_{4}\left(x_{\alpha}, t\right)=\operatorname{Re}\left[v_{4}\left(x_{\alpha}\right) e^{-i \omega t}\right],
\end{aligned}
$$

i.e., we assume that the system has reached steady state. The equations $(2.1)-(2.3)$ then become

$$
\begin{gathered}
h^{2}(\lambda+\mu) \operatorname{grad} \operatorname{div} v+\mu\left(h^{2} \Delta v-v-\operatorname{grad} v_{3}\right)-\operatorname{grad} v_{4}+\rho h^{2} \omega^{2} v=-m, \\
\mu\left[\Delta v_{3}+\operatorname{div} v\right]+\rho \omega^{2} v_{3}=-f_{3}, \\
\Delta v_{4}+\frac{i \omega}{K} v_{4}+i \omega \eta \alpha h^{2}(3 \lambda+2 \mu) \operatorname{div} v=-n,
\end{gathered}
$$

where $f_{3}$ and $n$ are the counterparts of $F_{3}$ and $N$, respectively, from relations 
similar to (2.5) and $v=v\left(x_{\alpha}\right)=\left(v_{1}, v_{2}, 0\right)^{\mathrm{T}}$. Equations (2.8)-(2.10) constitute the equations of steady thermoelastic oscillations for the bending of the plate.

3. Decomposition of a regular solution. The function $\phi$, defined in the domain $S$, will be called regular in $S$ if $\phi \in C^{2}(S) \cap C^{1}(S \cup \partial S)$. In what follows we consider regular solutions of the homogeneous system from (2.8)-(2.10), i.e.,

$$
\begin{gathered}
h^{2}(\lambda+\mu) \operatorname{grad} \operatorname{div} v+\mu\left(h^{2} \Delta v-v-\operatorname{grad} v_{3}\right)-\operatorname{grad} v_{4}+\rho h^{2} \omega^{2} v=0, \\
\mu\left[\Delta v_{3}+\operatorname{div} v\right]+\rho \omega^{2} v_{3}=0, \\
\Delta v_{4}+\frac{i \omega}{K} v_{4}+i \omega \eta \alpha h^{2}(3 \lambda+2 \mu) \operatorname{div} v=0 .
\end{gathered}
$$

This is without loss of generality since, as in [5], if $m, f_{3}$, and $n$ are sufficiently smooth, then the problem of solving (2.8)-(2.10) reduces to that of solving (3.1)-(3.3) and the construction of a particular solution in the form of a Newtonian potential.

As in [5] we can prove the following theorem.

THEOREM 3.1. Any regular solution of (3.1)-(3.3) is infinitely differentiable in its domain of regularity.

The main result of this section concerns the decomposition of any regular solution to (3.1)-(3.3) into vector fields representing a combination of damped and undamped waves. The complicated nature of the system (3.1)-(3.3) means that this decomposition is not as straightforward as in the case of classical elasticity [5].

THEOREM 3.2. In the domain of regularity any regular solution of (3.1)-(3.3) admits a representation of the form

$$
\begin{aligned}
U=\left(v, v_{3}, v_{4}\right) & =\left(v^{(1)}+v^{(2)}, v_{3}, v_{4}\right) \\
& =\sum_{i=1}^{5} \xi^{(i)},
\end{aligned}
$$

where

$$
\begin{gathered}
\left(\Delta+\lambda_{1}^{2}\right)\left(\Delta+\lambda_{2}^{2}\right)\left(\Delta+\lambda_{3}^{2}\right) v^{(1)}=0, \quad \operatorname{curl} v^{(1)}=0 ; \\
\left(\Delta+k_{3}^{2}\right) v^{(2)}=0 ; \quad \operatorname{div} v^{(2)}=0 ; \\
\left(\Delta+\lambda_{1}^{2}\right)\left(\Delta+\lambda_{2}^{2}\right)\left(\Delta+\lambda_{3}^{2}\right) v_{3}=0 ; \\
\left(\Delta+\lambda_{1}^{2}\right)\left(\Delta+\lambda_{2}^{2}\right)\left(\Delta+\lambda_{3}^{2}\right) v_{4}=0 ; \\
\left(\Delta+\lambda_{1}^{2}\right) \xi^{(1)}=0 ; \quad\left(\Delta+\lambda_{3}^{2}\right) \xi^{(2)}=0 ; \quad\left(\Delta+k_{3}^{2}\right) \xi^{(3)}=0 ; \\
\left(\Delta+\lambda_{2}^{2}\right) \xi^{(4)}=0 ; \quad\left(\Delta+\lambda_{3}^{2}\right) \xi^{(5)}=0 ; \\
k_{3}^{2}=\frac{\theta}{h^{2}}=\frac{k^{2} h^{2}-1}{h^{2}} ; \quad k^{2}=\frac{\rho \omega^{2}}{\mu} ;
\end{gathered}
$$


$-\lambda_{i}^{2}$ are the roots of

$$
\begin{gathered}
x^{3}+\left(\frac{i \omega}{K}+k_{1}^{2}+k_{2}^{2}+\frac{k_{1}^{2} k_{2}^{2}}{\theta_{2} \theta \mu k^{2}}\right) x^{2}+\left(\frac{i \omega}{K}\left(k_{1}^{2}+k_{2}^{2}\right)+k_{1}^{2} k_{2}^{2}\left(1+\frac{1}{\theta_{2} \theta \mu}\right)\right) x \\
+\frac{i \omega}{K} k_{1}^{2} k_{2}^{2}=0 ; \\
k_{1}^{2}+k_{2}^{2}=\frac{k^{2}(\lambda+3 \mu)}{\lambda+2 \mu}, \quad k_{1}^{2} k_{2}^{2}=\frac{\mu k^{2}\left(k^{2} h^{2}-1\right)}{h^{2}(\lambda+2 \mu)},
\end{gathered}
$$

and

Proof. Letting

$$
\theta_{2}=\left(i \omega \eta h^{2}(3 \lambda+2 \mu) \alpha\right)^{-1}
$$

$$
\begin{aligned}
& v^{(1)}=\frac{1}{\mu \theta}\left[-h^{2}(\lambda+2 \mu) \operatorname{grad} \operatorname{div} v+\mu \operatorname{grad} v_{3}+\operatorname{grad} v_{4}\right], \\
& v^{(2)}=\frac{h^{2}}{\theta} \text { curl curl } v,
\end{aligned}
$$

and proceeding as in [8], we obtain (3.4) and (3.6)-(3.9). Next, write

$$
V^{(1)}=\left(u^{(1)}, u_{3}, u_{4}\right), \quad V^{(2)}=\left(u^{(2)}, w_{3}, w_{4}\right), \quad V^{(3)}=\left(v^{(2)}, 0,0\right),
$$

where

$$
\begin{array}{rlrl}
u^{(1)} & =\frac{\left(\Delta+\lambda_{2}^{2}\right) v^{(1)}}{\lambda_{2}^{2}-\lambda_{1}^{2}}, & u^{(2)}=\frac{\left(\Delta+\lambda_{1}^{2}\right) v^{(1)}}{\lambda_{1}^{2}-\lambda_{2}^{2}}, & u_{3}=\frac{\left(\Delta+\lambda_{2}^{2}\right) v_{3}}{\lambda_{2}^{2}-\lambda_{1}^{2}}, \\
u_{4}=\frac{\left(\Delta+\lambda_{2}^{2}\right) v_{4}}{\lambda_{2}^{2}-\lambda_{1}^{2}}, & w_{3}=\frac{\left(\Delta+\lambda_{1}^{2}\right) v_{3}}{\lambda_{1}^{2}-\lambda_{2}^{2}}, & w_{4}=\frac{\left(\Delta+\lambda_{1}^{2}\right) v_{4}}{\lambda_{1}^{2}-\lambda_{2}^{2}}
\end{array}
$$

Then,

$$
\left(\Delta+\lambda_{1}^{2}\right)\left(\Delta+\lambda_{3}^{2}\right) V^{(1)}=0, \quad\left(\Delta+\lambda_{2}^{2}\right)\left(\Delta+\lambda_{3}^{2}\right) V^{(2)}=0, \quad\left(\Delta+k_{3}^{2}\right) V^{(3)}=0,
$$

and

$$
U=\left(v^{(1)}+v^{(2)}, v_{3}, v_{4}\right)=\sum_{i=1}^{3} V^{(i)}
$$

Now, if

$$
\begin{gathered}
\xi^{(1)}=\frac{\left(\Delta+\lambda_{3}^{2}\right) V^{(1)}}{\lambda_{3}^{2}-\lambda_{1}^{2}}, \quad \xi^{(2)}=\frac{\left(\Delta+\lambda_{1}^{2}\right) V^{(1)}}{\lambda_{1}^{2}-\lambda_{3}^{2}}, \quad \xi^{(3)}=V^{(3)}, \\
\xi^{(4)}=\frac{\left(\Delta+\lambda_{3}^{2}\right) V^{(2)}}{\lambda_{3}^{2}-\lambda_{1}^{2}}, \quad \xi^{(5)}=\frac{\left(\Delta+\lambda_{2}^{2}\right) V^{(2)}}{\lambda_{2}^{2}-\lambda_{3}^{2}}
\end{gathered}
$$

from (3.12) we obtain (3.5) and from (3.6)-(3.9), we obtain (3.10) and (3.11).

Note 3.3. If we impose the condition $\omega>C_{t} / h$ [8] where $C_{t}^{2}=\mu / \rho$ is the speed of transverse waves in an infinite elastic medium, then $k_{i}^{2}>0$ and (3.5) is seen to represent the sum of five waves, four moving with different speeds and all damped except for the shear wave, which is unaffected by the thermal effects. 
4. Radiation conditions. The steady-state system (3.1)-(3.3) is more amenable to mathematical treatment than its full dynamic counterpart (2.1)-(2.3). However, associated boundary value problems for (3.1)-(3.3) lose the uniqueness property proved for the corresponding initial-boundary value problems for $(2.1)-(2.3)$ [4]. This loss of uniqueness can be explained by the presence of eigenfrequencies in (3.1)-(3.3). In bounded regions there is no unique solution for a discrete spectrum of eigenfrequencies. In the case of unbounded regions (exterior boundary value problems) uniqueness can be secured by requiring that any solution of (3.1)-(3.3) satisfy radiation conditions similar to the Sommerfeld radiation condition for the wave equation [3].

Let $S^{+} \equiv S$ and $S^{-} \equiv \mathbf{R}^{2} \backslash\left(S^{+} \cup \partial S\right)$.

Definition 4.1. Let $x \in S^{-}$. Then any solution $\left(v, v_{3}, v_{4}\right)$ of $(3.1)-(3.3)$ is said to satisfy a radiation condition if, as $|x|=R \rightarrow \infty$,

$$
\begin{array}{rlrl}
v^{(1)}(x) & =o\left(R^{-\frac{1}{2}}\right), & & \frac{\partial v^{(1)}}{\partial x_{\alpha}}(x)=O\left(R^{-1}\right) ; \\
v^{(2)}(x)=O\left(R^{-\frac{1}{2}}\right), & & \frac{\partial v^{(2)}}{\partial R}(x)-i k_{3} v^{(2)}(x)=o\left(R^{-\frac{1}{2}}\right) ; \\
v_{3}(x)=o\left(R^{-\frac{1}{2}}\right), & & \frac{\partial v_{3}}{\partial x_{\alpha}}(x)=O\left(R^{-1}\right) ; \\
v_{4}(x)=o\left(R^{-\frac{1}{2}}\right), & & \frac{\partial v_{4}}{\partial x_{\alpha}}(x)=O\left(R^{-1}\right) .
\end{array}
$$

Note 4.2. Only $v^{(2)}$ satisfies a vector Helmholtz equation. Hence, only $v^{(2)}$ has a Sommerfeld radiation condition.

5. Uniqueness Theorem. As in [4] we represent stress at a point $x$ by the $(3 \times 1)$ matrix

$$
P(\partial x, n(x)) W(x)
$$

where $P(\partial x, n(x))$ is the $(3 \times 4)$-matrix differential operator defined by

$$
\begin{aligned}
& P_{i j}(\partial x, n(x))=T_{i j}(\partial x, n(x)), \\
& P_{\alpha 4}(\partial x, n(x))=-n_{\alpha}(x), \\
& P_{34}(\partial x, n(x))=0,
\end{aligned}
$$

$T_{i j}$ are the elements of the stress operator in the elastostatic case [6], $n=\left(n_{1}, n_{2}, 0\right)^{\mathrm{T}}$ an arbitrary direction in the plate's middle surface, and $W(x)=\left(v_{1}, v_{2}, v_{3}, v_{4}\right)^{\mathrm{T}}$. Write (3.1)-(3.3) in the form

$$
\begin{gathered}
{\left[A(\partial x)+\left(\begin{array}{ccc}
\rho h^{2} \omega^{2} & 0 & 0 \\
0 & \rho h^{2} \omega^{2} & 0 \\
0 & 0 & \rho \omega^{2}
\end{array}\right)\right] u(x)-\operatorname{grad} v_{4}=0,} \\
\Delta v_{4}+\frac{i \omega}{K} v_{4}+\frac{1}{\theta_{2}} \operatorname{div} v=0
\end{gathered}
$$


where here, $u(x)=\left(v_{1}, v_{2}, v_{3}\right)^{\mathrm{T}}$ and $A(\partial x)$ is the $(3 \times 3)$-matrix from elastostatics [6], i.e., $A(\partial x)=A\left(\partial / \partial x_{\gamma}\right)=A\left(\xi_{\gamma}\right)$ is the matrix

$$
\left(\begin{array}{ccc}
h^{2} \mu \Delta+h^{2}(\lambda+\mu) \xi_{1}^{2}-\mu & h^{2}(\lambda+\mu) \xi_{1} \xi_{2} & -\mu \xi_{1} \\
h^{2}(\lambda+\mu) \xi_{1} \xi_{2} & h^{2} \mu \Delta+h^{2}(\lambda+\mu) \xi_{2}^{2}-\mu & -\mu \xi_{2} \\
\mu \xi_{1} & \mu \xi_{2} & \mu \Delta
\end{array}\right) .
$$

Denoting the complex conjugate of $u$ by $\bar{u}$ and using the relation [6]

$$
\int_{S^{+}}\left[\bar{u}^{\mathrm{T}} A(\partial x) u+2 E(\bar{u}, u)\right] d \sigma=\int_{\partial S} \bar{u}^{\mathrm{T}}(T u) d S,
$$

we can write

$$
\begin{gathered}
\int_{S^{+}}\left[\bar{u} A(\partial x) u-\bar{u}^{\mathrm{T}} \operatorname{grad} v_{4}+2 E(\bar{u}, u)-v_{4} \operatorname{div} \bar{u}\right] d \sigma \\
=\int_{\partial S}\left[\bar{u}^{\mathrm{T}}(T u)-n \cdot v_{4} \bar{u}\right] d S .
\end{gathered}
$$

From $(5.2)_{2}$ and the identity

$$
\int_{S^{+}}\left[v_{4} \Delta \bar{v}_{4}+\operatorname{grad} v_{4} \cdot \operatorname{grad} \bar{v}_{4}\right] d \sigma=\int_{\partial S} v_{4} \frac{\partial \bar{v}_{4}}{\partial n} d S
$$

we obtain

$$
\frac{i \omega}{K} \int_{S^{+}}\left|v_{4}\right|^{2} d \sigma+\int_{S^{+}}\left|\operatorname{grad} v_{4}\right|^{2} d \sigma-\int_{\partial S} v_{4} \frac{\partial \bar{v}_{4}}{\partial n} d S=-\frac{1}{\theta_{2}} \int_{S^{+}} v_{4} \operatorname{div} \bar{v} d \sigma .
$$

From $(5.2)_{1},(5.3)$, and (5.4) we have

$$
\begin{aligned}
\int_{S^{+}}\left[-\rho \omega^{2}\left(h^{2}\left|v_{1}\right|^{2}+h^{2}\left|v_{2}\right|^{2}+\left|v_{3}\right|^{2}\right)+2\right. & \left.E(\bar{u}, u)+\frac{i \omega}{K} \theta_{2}\left|v_{4}\right|^{2}+\theta_{2}\left|\operatorname{grad} v_{4}\right|^{2}\right] d \sigma \\
= & \int_{\partial S} \bar{u}^{\mathrm{T}} P W d S+\theta_{2} \int_{\partial S} v_{4} \frac{\partial \bar{v}_{4}}{\partial n} d S .
\end{aligned}
$$

Taking the complex conjugate of (5.5), subtracting this from (5.5), and noting that $E(\bar{u}, u)=E(u, \bar{u})[6]$, we obtain

$$
2 \theta_{2} \int_{S^{+}}\left|\operatorname{grad} v_{4}\right|^{2} d \sigma=\int_{\partial S}\left[\bar{u}^{\mathrm{T}} P W-u^{\mathrm{T}} P \bar{W}+\theta_{2}\left(v_{4} \frac{\partial \bar{v}_{4}}{\partial n}+\bar{v}_{4} \frac{\partial v_{4}}{\partial n}\right)\right] d S .
$$

Equation (5.6) is instrumental in proving the following uniqueness theorem.

Theorem 5.1. The solution, regular in $S^{-}$, of Eqs. (3.1)-(3.3) is identically zero if it satisfies a radiation condition, $\omega>C_{t} / h$, and one of the following boundary conditions on $\partial S$ :

(i) $W=0$,

(ii) $P W=0, \frac{\partial v_{4}}{\partial n}=0$,

(iii) $u=0, \frac{\partial v_{4}}{\partial n}=0$,

(iv) $P W=0, v_{4}=0$,

(v) $\partial S=\bigcup_{k=1}^{4} \partial S_{k}$ with the $k$ th boundary condition above given on $\partial S_{k}$. 
Proof. Let $\partial K_{R}$ be the circumference of a circle $K_{R}$, radius $R$, sufficiently large to enclose $\partial S$. Applying (5.6) in the domain $K_{R} \cap S^{-}$and the boundary conditions give

$$
\begin{aligned}
& 2 \theta_{2} \int_{K_{R} \cap S^{-}}\left|\operatorname{grad} v_{4}\right|^{2} d \sigma \\
& \quad=\int_{\partial K_{R}}\left[\bar{u}^{\mathrm{T}} P W-u^{\mathrm{T}} P \bar{W}+\theta_{2}\left(v_{4} \frac{\partial \bar{v}_{4}}{\partial n}+\bar{v}_{4} \frac{\partial v_{4}}{\partial n}\right)\right] d S .
\end{aligned}
$$

The right-hand side of (5.7) can be written as

$$
\begin{gathered}
\int_{\partial K_{R}}\left[\bar{u}^{\mathrm{T}} T u-\bar{u}^{\mathrm{T}} n v_{4}-u^{\mathrm{T}} T \bar{u}+u^{\mathrm{T}} n \bar{v}_{4}+\theta_{2}\left(v_{4} \frac{\partial \bar{v}_{4}}{\partial n}+\bar{v}_{4} \frac{\partial v_{4}}{\partial n}\right)\right] d S \\
=\int_{\partial K_{R}}\left[\bar{v}^{\mathrm{T}} T v-v^{\mathrm{T}} T \bar{v}+\mu\left(\bar{v}_{3} v^{\mathrm{T}} n-v_{3} \bar{v}^{\mathrm{T}} n-v_{3} \frac{\partial \bar{v}_{3}}{\partial n}+\bar{v}_{3} \frac{\partial v_{3}}{\partial n}\right)\right. \\
\left.-\bar{u}^{\mathrm{T}} n v_{4}+u^{\mathrm{T}} n \bar{v}_{4}+\theta_{2}\left(v_{4} \frac{\partial \bar{v}_{4}}{\partial n}+\bar{v}_{4} \frac{\partial v_{4}}{\partial n}\right)\right] d S .
\end{gathered}
$$

Writing $v=v^{(1)}+v^{(2)}$ and applying the radiation condition, (5.8) becomes

$$
\begin{aligned}
\int_{\partial K_{R}} & {\left[\left(\bar{v}^{(2)}\right)^{\mathrm{T}} T v^{(2)}-\left(v^{(2)}\right)^{\mathrm{T}} T \bar{v}^{(2)}\right] d S+o(1) } \\
= & \int_{\partial K_{R}}\left\{\left(\bar{v}^{(2)}\right)^{\mathrm{T}}\left[T v^{(2)}-i h^{2} \mu k_{3} v^{(2)}\right]-v^{(2)}\left[T \bar{v}^{(2)}+i h^{2} \mu k_{3} \bar{v}^{(2)}\right]\right. \\
& \left.+2 i h^{2} \mu k_{3}\left|v^{(2)}\right|^{2}\right\} d S .
\end{aligned}
$$

From [7],

$$
T v^{(2)}-i h^{2} \mu k_{3} v^{(2)}=o\left(R^{-1 / 2}\right) .
$$

Letting $R \rightarrow \infty$ in (5.7) and using (5.9), (5.10), we obtain

$$
i \theta_{2} \int_{S^{-}}\left|\operatorname{grad} v_{4}\right|^{2} d \sigma+h^{2} \mu k_{3} \lim _{R \rightarrow \infty} \int_{\partial K_{R}}\left|v^{(2)}\right|^{2} d S=0 .
$$

The conditions (2.4) and $\omega>C_{t} / h$ ensure that both $i \theta_{2}$ and $h^{2} \mu k_{3}$ are positive. Hence (5.11) yields

$$
\lim _{R \rightarrow \infty} \int_{\partial K_{R}}\left|v^{(2)}\right|^{2} d S=0 ; \quad \int_{S^{-}}\left|\operatorname{grad} v_{4}\right|^{2} d \sigma=0 .
$$

$(5.12)_{1},(3.7)_{1}$ together with Rellich's Lemma for the Helmholtz equation [3] yield

$$
v^{(2)}=0 \text { in } S^{-} \text {. }
$$

$(5.12)_{2}$ yields $v_{4}=c=$ constant in $S^{-}$. However, the imposed radiation condition means that $c=0$ so that

$$
v_{4}=0 \text { in } S^{-} .
$$

From (3.3), (3.7) $)_{2}$, and (5.14) we have

$$
\operatorname{div} v=\operatorname{div} v^{(1)}=0 \quad \text { in } S^{-} .
$$


(3.2) and (5.15) now give

$$
\left(\Delta+k^{2}\right) v_{3}=0 \quad \text { in } S^{-}, \quad k^{2}=\frac{\rho \omega^{2}}{\mu}>0 .
$$

The radiation condition implies that

$$
\lim _{R \rightarrow \infty} \int_{\partial K_{R}}\left|v_{3}\right|^{2} d S=0 .
$$

(5.16) and (5.17) now yield

$$
v_{3}=0 \text { in } S^{-}
$$

on application of Rellich's Lemma. Finally, from the proof of Theorem 3.2,

$$
v^{(1)}=\frac{1}{\mu \theta}\left[-h^{2}(\lambda+2 \mu) \operatorname{grad} \operatorname{div} v+\mu \operatorname{grad} v_{3}+\operatorname{grad} v_{4}\right] \text {. }
$$

However, (5.14), (5.15), and (5.18) imply that

$$
v^{(1)}=0 \text { in } S^{-} \text {. }
$$

Hence, from (5.13), (5.14), (5.18), and (5.19) it follows that $v=v^{(1)}+v^{(2)}=0$, $v_{3}=0$, and $v_{4}=0$, i.e., $v_{1}=v_{2}=v_{3}=v_{4}=0$ in $S^{-}$.

Note 5.2. Similar results can be proved for the micropolar thermoelastic plate model introduced in [4].

\section{REFERENCES}

[1] A. C. Eringen and E. S. Suhubi, Elastodynamics, Volume II, Academic Press, New York, 1975

[2] J. A. Hudson, The Excitation and Propagation of Elastic Waves, Cambridge University Press, Cambridge, 1980

[3] D. Colton and R. Kress, Intregral Equation Methods in Scattering Theory, Wiley, New York, 1983

[4] P. Schiavone and R. J. Tait, Thermal effects in Mindlin-type plates, Quart. J. Mech. Appl. Math. 46, 27-39 (1993)

[5] V. D. Kupradze, T. G. Gegelia, M. O. Basheleǐshvili, and T. V. Burchuladze, Three-Dimensional Problems of the Mathematical Theory of Elasticity and Thermoelasticity, North-Holland Publ. Co., Amsterdam, 1979

[6] C. Constanda, A Mathematical Analysis of Bending of Plates with Transverse Shear Deformation, Pitman Research Notes in Mathematics Series, No. 215, 1990

[7] C. Constanda and P. Schiavone, Flexural waves in Mindlin-type plates, ZAMM 74, 492-493 (1994)

[8] P. Schiavone and C. Constanda, Oscillation problems in thin plates with transverse shear deformation, SIAM J. Appl. Math. 53, 1253-1263 (1993) 